\title{
Construction and Tests of Resistive Plate Chambers for the Upgrade of the CMS Endcap System
}

\author{
L. M. Pant on behalf of the CMS collaboration \\ Nuclear Physics Division \\ Bhabha Atomic Research Centre, Mumbai 400085, India \\ E-mail : lmpant@barc.gov.in, lalit_pant@rediffmail.com
}

\begin{abstract}
The CMS muon endcap system presently comprises three disks of RPCs, with a fourth disk under preparation with detectors and associated services. This station will be installed in the first long shutdown of LHC during 2013-2014. In this paper we describe the RPCs with new construction and test procedures for this upgrade, based on lessons learnt from the earlier work. We report on construction and tests performed after the assembly of first two prototypes on the standardisation of leak and spacer tests for the bakelite gas-gaps, and on the new design for the $\mathrm{Cu}$ cooling system.
\end{abstract}

XI workshop on Resistive Plate Chambers and Related Detectors (RPC2012)

INFN-Laboratori Nazionali di Frascati, Italy

February 5-10, 2012 


\section{Introduction}

The muon trigger for the CMS experiment at the LHC facility, is designed to have four layers of Resistive Plate Chambers (RPCs) in the forward region $(1.2<\eta<1.6)$ [1]. In the present phase, the experiment runs with three layers of RPCs in the end-caps. The end-caps consist of three trapezoidal bakelite gas-gaps configured as a double gap RPC [2], with each RPC having a segmented readout strip along $\eta$, called as $\eta$ partitions. In order to increase the L1 trigger efficiency, it is proposed to install the fourth end-cap layer during the long shutdown (2013-2014), after which LHC will run with its full designed luminosity.

With the experience gained during the operation of RPCs in the present set-up, the construction procedure of RPCs has been improved, keeping backward compatibility with the presently installed RPCs. As in the past, the fourth layer will consist of 2 rings denominated as RE4/2 and RE4/3, each having 36 chambers. Both end-caps will be instrumented for a total of 144 chambers with another 56 chambers as spares, adding to 200 chambers for the RPC upgrade project, which then fully restores the TDR version of CMS with RE4 RPCs.

\section{RE4 prototype assembly}

A pre-production run, prior to the actual mass production of gas-gaps for the RPC upgrade was planned in March/April 2011 and about 18 gas-gaps for the two rings (RE4/2 and RE4/3) were sent to CERN for their evaluation. The prototype asembly for RE4 was thus launched with the arrival of fresh set of gas-gaps from Korean DEtector Lab, KODEL, South Korea, mechanics from China and the improved $\mathrm{Cu}$ cooling system from India. Two prototypes, one for each ring were assembled. Figure 1, shows a fully assembled RPC for the RE4/2 ring under test in a dedicated laboratory at CERN and its corresponding efficiency plot for one of the $\eta$ partitions (A1), at different threshold settings.
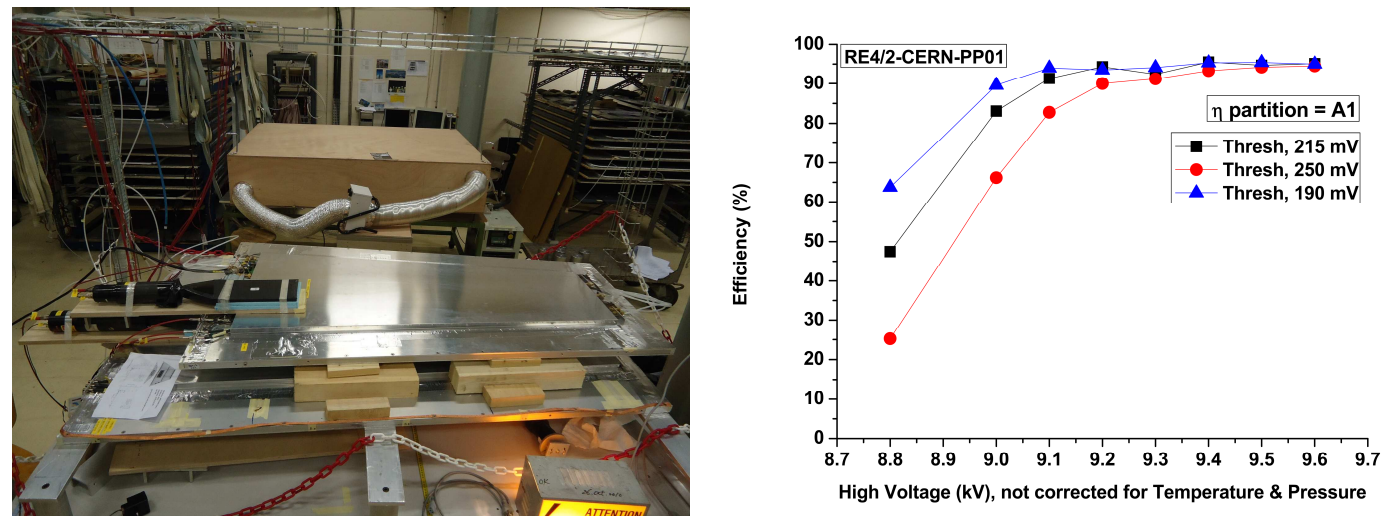

Fig. 1, RE4/2 prototype under tests (left) with efficiency plot (right) for different threshold settings 
The above quality test validated the entire construction chain and the compatibility of various components used to build the chamber, like the bakelite in terms of surface smoothness and its bulk resistivity. It also validated the gas-gap fabrication procedures in terms of its mechanical and electrical properties, gluing of gas-gaps, bonding of spacers. Finally the measured cosmic efficiencies did certify the behaviour of gas-gaps coupled to the front end electronics, matching the specifications set by CMS. During the pre-production run all the necessary mechanical components were revalidated and the technical specifications and quality assurance and quality control, QA/QC protocols were formalized. For the RPC upgrade project, 660 gas-gaps will be produced at KODEL, with a production rate of 3 gaps/day, thereby needing 11 months for the entire gap production.

\section{Leak and spacer test for the gas-gaps}

The trapezoidal gas-gaps have nozzles in the four corners for gas flow. The $2 \mathrm{~mm}$ gap uniformity is maintained through a grid $(10 \mathrm{~cm} \times 10 \mathrm{~cm})$ of button spacers (of height uniformity within $20 \mu \mathrm{m}$ ) between the bakelite sheets. The $20 \mu \mathrm{m}$ precision ensure that the gap remains uniform over the entire area of the gas-gap providing a uniform electric field. Since the gas-gaps are transported through a large distance via air freight and then handled at airports before delivery to the respective assembly sites, it might be possible that because of mishandling some of the button spacers get popped up. These spacers could also get popped up because of insufficient bonding of the glue at the fabrication site, though sufficient care is taken to keep the spacers pressed, guaranteed by water column, for 14 hours during curing. In either case a gasgap with a popped spacer cannot be used and is to be rejected.

In order to certify these gas-gaps a new system has been designed where the gas-gaps on arrival from KODEL, undergo a leak and spacer test. The gas-gaps are pressurised at 20 mbar above atmospheric pressure with Argon gas and the pressure is recorded digitally through a transducer (Sensor Technics - CTE7000) with a 20 bit ADC. The QA/QC "as prescribed by the CMS experiment for the leak test" needs the gap to remain pressurised at 20 mbar for 10 minutes and the $\mathrm{dP} / \mathrm{dt}$ for each gas-gap is measured. Then, the overpressure is reduced to 3 mbar, which emulates the situation in the CMS cavern, for the next 10 minutes. The $\mathrm{dP} / \mathrm{dt}$ is measured again at 3 mbar and then the overpressure is released to zero. A plot of the measured $\mathrm{dP} / \mathrm{dt}$ is shown in the left panel of Fig.2 for the RE4/2-bottom gas-gap with 102 spacers. The insert in the left panel corresponds to the acceptable leak rate $\left(2.3 \times 10^{-4} \mathrm{mbar} \times \mathrm{litre} / \mathrm{s}\right)$ from the particular gas-gap. For spacer test, a template with known spacer position is placed over the gap and each spacer position is pressed to record the fluctuations in the pressure. For intact spacers, the fluctuations in the pressure are in the range of $\pm 0.5 \mathrm{mbar}$, whereas for the same pressure applied in the non-spacer region, resembling a popped up spacer, corresponds to large fluctuations ( \pm 1 mbar and beyond), as shown in the right panel of Fig. 2 . 

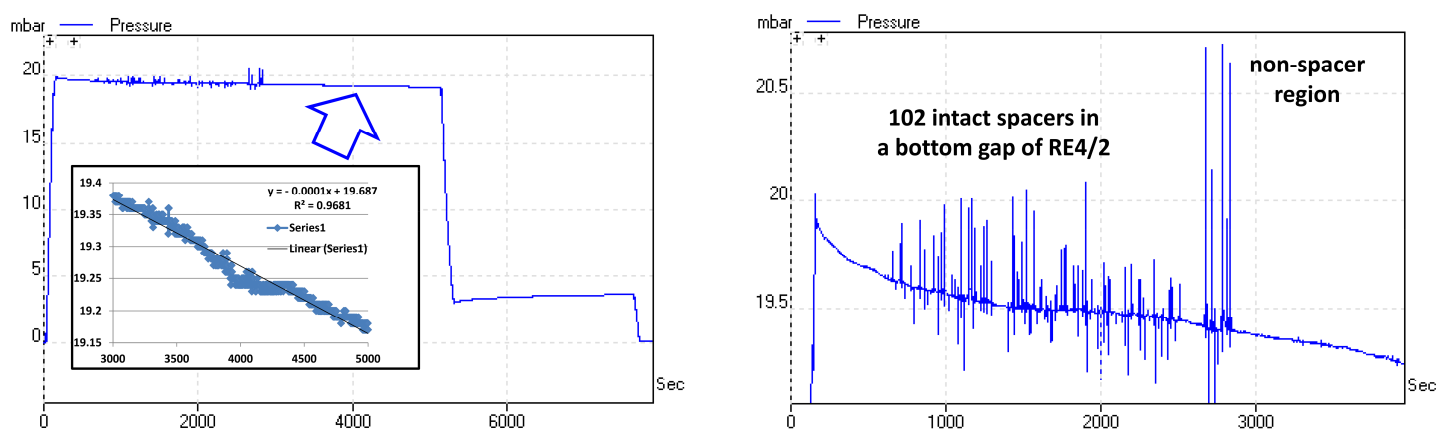

Fig. 2, Gas-gaps pressurised to 20 mbar and 3 mbar of overpressure to calculate the leak rate (left panel) and spacer test for gas-gaps showing acceptable pressure fluctuations ( \pm 0.5 mbar) for intact spacers (right panel)

\section{Improved Cu cooling system for the RE4-RPCs}

With the experience gained with the installed RPCs in the existing end-caps, the $\mathrm{Cu}$ cooling system for the RPCs was redesigned. In the new design, the area of $\mathrm{Cu}$ plates has been increased optimally with water flowing through $\mathrm{Cu}$ pipes, which run parallely. The earlier installed, $8 \mathrm{~mm} \phi$ brass unions with a single ferrule, have been replaced with SS double ferrules to ensure that there is no water leakage at the coupling junctions. Improvement in the cooling system had to be introduced for RE4, due to its particular position facing the electronics of the other muon system based on Cathode Strip Chambers. The new cooling system is envisaged also for RPC that will replace malfunctioning chambers in the other layers, so the design includes backward compatibility.

A prototype of the $\mathrm{Cu}$ cooling system was fabricated in BARC, Mumbai, meeting the required specifications and was dispatched to CERN during the pre-production run. The $\mathrm{Cu}$ cooling system was fabricated with Deoxidized High Phosphorus (DHP) semi hard Cu pipes (8 $\mathrm{mm}$ OD and $6 \mathrm{~mm} \mathrm{ID)}$ and $\mathrm{Cu}$ sheets with $1 \mathrm{~mm}$ thickness. The DHP Copper is a commercially available material of pure copper, which has been deoxidized with phosphorus, leaving relatively high residual phosphorus content. This copper has a lower electrical conductivity and is used where there is need for heat transfer and electrical properties are not important. The $\mathrm{Cu}$ cooling system will have chilled water at $19^{\circ} \mathrm{C}$ running at a pressure of $2 \mathrm{bar}$, in order to cool the electronics and the body of RPCs through the aluminum honey comb panels on to which it is mounted. A typical $\mathrm{Cu}$ cooling system for RE4/3 type RPC is shown in Fig. 3. The $\mathrm{Cu}$ pipes were soldered $\left(\sim 200{ }^{\circ} \mathrm{C}\right)$ to the $\mathrm{Cu}$ plates with soldering material having a composition of $\mathrm{Sn}$ : $\mathrm{Ag}: \mathrm{Pb}:: 62: 2: 36$. After complete assembly, the $\mathrm{Cu}$ cooling systems are tested for any possible leakage with Argon gas at 20 bar of pressure. 


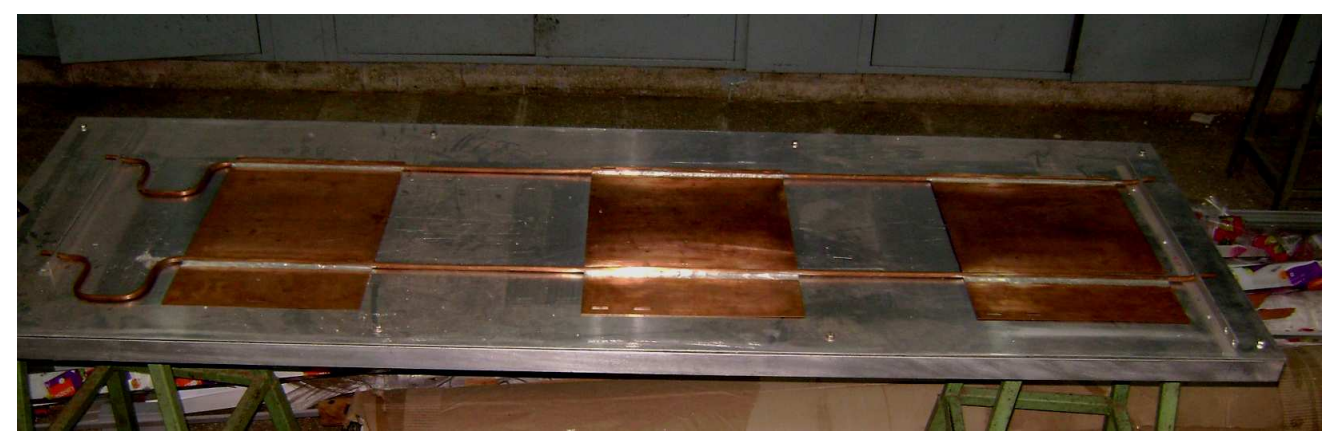

Fig. 3, Cu cooling assembly for a typical RE4/3 RPC

\section{Chamber assembly sites and delivery schedules}

For the start up it is proposed to build 50 chambers each in India (RE4/2), and University of Ghent, Belgium (RE4/3) and the remaining (50 chambers of RE4/2 and 50 chambers of RE4/3) at CERN. In India, two institutes are collaborating jointly for the project, namely - Nuclear Physics Division-BARC, Mumbai and Panjab University, Chandigarh. CERN site will coordinate the logistics \& setting up protocols for QA/QC. Construction data base has been implemented for QC from chamber components (bakelite, gaps, electronic etc.) to final chamber supermodule assembly. At the time of writing this paper, the relevant mechanics and read out planes have arrived from China at the respective assembly sites for all the 200 chambers and the RPC assembly work is expected to begin soon after the arrival of first batch of gas-gaps at the assembly sites. Gas-gaps will be produced at a rate of approximately 60 gaps per month at KODEL, and will then be dispatched to the three sites.

Figure 4, shows the three assembly sites respectively at Mumbai, CERN and Ghent. The foreseen chamber production rate is three RPCs per month in Mumbai, five RPCs per month at Ghent and ten RPCs per month at CERN, given the available logistics and infrastructure in these sites. It is proposed to have the first ten chambers from each site by October 2012, additional chambers for disk1 by May 2013, all chambers for disk2 by end of 2013 and the remaining spares by spring 2014. All the assembly sites are ready with their respective cosmic hodoscopes, VME based DAQs and QA/QC procedures for the launch of production of the first ten chambers, each, from May/June 2012 onwards. Instrumentation for monitoring of the environmental pressure, temperature, and relative humidity to run the HV scripts and for ramping of gas-gaps for dark current measurements have been implemented in all the three sites. The development of software tools for offline analysis and characterization of RPCs is ongoing. 

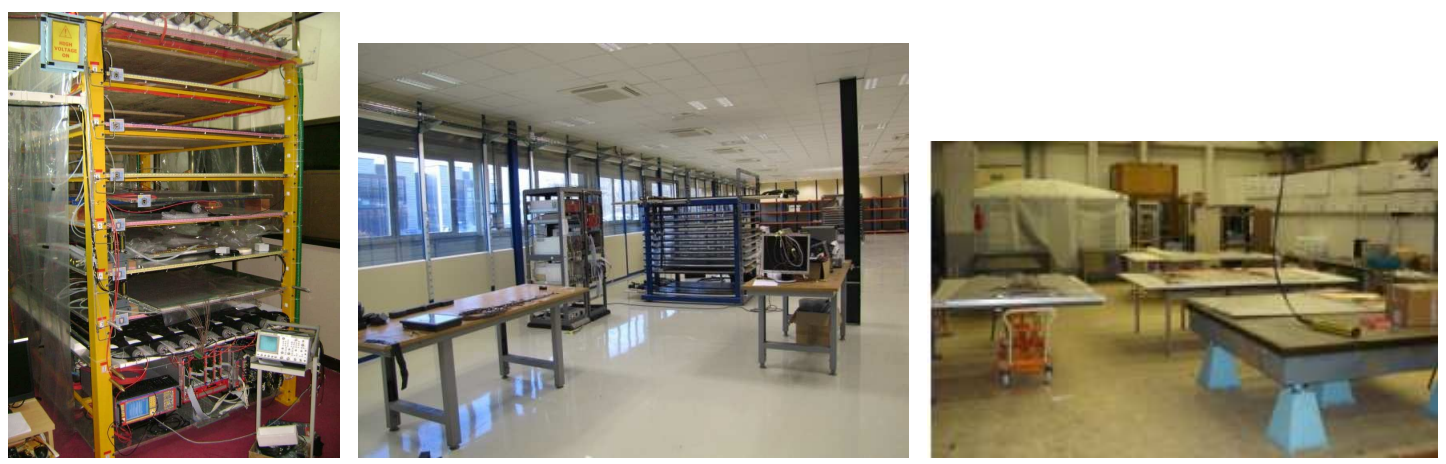

Fig. 4, Cosmic hodoscope at NPD-BARC, Mumbai (left), CERN-904 site (middle) and Ghent site (right)

\section{Acknowledgements}

We are thankful to the Indian agencies, Department of Atomic Energy and Department of Science and Technology for funding the Indian contribution for the upgrade project, the RPC end-cap group at CERN for providing all the logistical support and this article being blessed by CMS RPC EB.

\section{References}

[1] CMS Collaboration, The Muon Project, Technical Design Report, CERN/LHCC 97-32, December 1997

[2] L. M. Pant, Nuclear Instr. \& Methods in Physics Research A 661 (2012) S34-S36 